\title{
Sensitisation to Mallory bodies (alcoholic hyalin) in alcoholic hepatitis
}

\author{
STELLA M TRIGGS, PR MILLS, AND RNM MACSWEEN \\ From the University Department of Pathology and Department of Medicine, The Western Infirmary, \\ Glasgow G11 6NT, UK
}

SUMMARY Using a leucocyte migration inhibition test sensitisation to Mallory bodies (alcoholic hyalin) was found in a statistically significant $41 \%$ of 17 patients with alcoholic hepatitis. Patients with alcohol-induced fatty liver and cirrhosis did not demonstrate sensitisation. Mallory bodies are a characteristic feature of alcohol-induced liver damage, and immunological sensitisation to them might lead to liver cell death and progression of the hepatitis process.

The hepatotoxic effects of alcohol in man are now well recognised and result in the spectrum of alcoholic liver disease, viz, fatty liver, alcoholic hepatitis, and cirrhosis. The clinical and histological aspects of these have been the subject of recent reviews. ${ }^{12}$ These hepatic effects are in part related to the amount of alcohol ingested and to the duration of alcohol abuse, ${ }^{3}$ but there is convincing evidence that additional host factors determine susceptibility to both the initial alcohol-induced liver damage and the progression to chronicity. Whereas the majority of those who drink alcohol to excess develop a fatty liver, it seems that only $30-40 \%$ develop alcoholic hepatitis, and of these only a further third (ie, 10-15\% of the 'heavy-drinking population') develop cirrhosis. ${ }^{4}$ These host factors remain to be defined, and as yet no consistent phenotypic markers have been reported.

Leevy and his co-workers ${ }^{5}$ have suggested that immunological mechanisms may be of importance in the chronicity and progression of the liver lesions. In particular, these workers provided evidence that sensitisation to alcoholic hyalin or Mallory bodies, a characteristic morphological feature in alcoholic hepatitis, may be of pathogenic significance in alcoholic hepatitis and cirrhosis. ${ }^{6-8}$ In the present paper we report studies in which isolated Mallory bodies have been used as antigen in migration inhibition studies with peripheral blood leucocytes from patients with histologically defined alcoholinduced liver disease. The results indicate that a proportion of patients, particularly with alcoholic hepatitis, have demonstrable in vitro sensitisation to this antigen.

Accepted for publication 5 June 1980

\section{Patients and methods}

\section{CLINICAL STUDIES}

The 38 patients investigated all gave a clinical history of prolonged alcohol abuse and all had biopsy-proven evidence of liver disease. They were subdivided on the basis of the histological findings into those with fatty liver (6 patients), alcoholic hepatitis (17 patients), and alcoholic cirrhosis (15 patients), the histological diagnosis being made by one observer (RNMMacS) without knowledge of the in vitro laboratory studies. The morphological severity of the alcoholic hepatitis was assessed on an arbitrary 1 to $4+$ scale. The control subjects comprised 30 normal healthy members of the medical and laboratory staff, none of whom was given to alcohol abuse. Patients in the alcoholic hepatitis group were assessed clinically using six criteria, which in three retrospective studies had been shown to correlate with a fatal outcome. ${ }^{9-11}$ From figures given in these series of patients arbitrary values were selected to divide mild from severe cases using the following scoring system:

Bilirubin $>85 \mu \mathrm{mol} / 1$ Peripheral blood leucocyte count $>10000 \mathrm{~mm}^{3}\left(10 \times 10^{9} / \mathrm{l}\right) \quad 1$

Blood urea $>20000 \mathrm{~mm}^{3}\left(20 \times 10^{9} / \mathrm{l}\right)$

Hepatic encephalopathy $>7 \mu \mathrm{mol} / 1$

Prothrombin time $>4$ s prolonged Ascites

The clinical severity was thus assessed as mild (score 0-2), moderate (3-4), or severe (5-8).

LABORATORY ST UDIES

Mallory bodies were isolated from livers collected 
from patients with alcoholic hepatitis. The isolation procedure was the two-phase polymer system method of Okamura and his colleagues, ${ }^{12}$ modified in that before the polymer separation the following additional step was introduced:13 tissue homogenates were digested at $37^{\circ} \mathrm{C}$ for $5-24$ hours in tris buffer $(0.05 \mathrm{M}, \mathrm{pH} 7.5)$ containing $0.1 \%$ DNAase (Sigma), $0.1 \%$ clostridia collagenase (Worthington Enzymes), $0.36 \mathrm{mM} \mathrm{CaCl}_{2}, 0.1 \mathrm{~mm} \mathrm{MgSO} 4$, mycostatin (50 units $/ \mathrm{ml})$, and tetracycline $(0 \cdot 25 \mathrm{mg} / \mathrm{ml})$. The final product was examined by light and electron microscopy and confirmed the presence of bodies with the tinctorial properties and the ultrastructural features of Mallory bodies. Livers from patients with no evidence of liver disease were treated in an identical way and the product was used as control antigen. The protein content was assessed using the Folin reaction. The antigen was sonicated, resuspended in saline at a concentration of $1 \mathrm{mg}$ protein $/ \mathrm{ml}$, and stored at $-20^{\circ} \mathrm{C}$ for use in the migration inhibition tests.

Leucocytes were separated from $30 \mathrm{ml}$ of heparinised blood by sedimentation for 1 hour at $37^{\circ} \mathrm{C}$ with $5 \mathrm{ml}$ Plasmagel (Laboratoire Roger Bellon, Neuilly, France). The white-cell-rich plasma was then centrifuged at $400 \mathrm{~g}$ for 5 minutes, washed twice in phosphate buffered saline $(0 \cdot 1 \mathrm{M}, \mathrm{pH} 7 \cdot 4)$, and resuspended in Eagle's medium $+10 \%$ fetal calf serum (FCS) at a concentration of $1 \times 10^{8}$ cells $/ \mathrm{ml}$. Two variants of the migration inhibition test were used:

(1) preincubation, in which $200 \mu$ l of the leucocyte suspension were preincubated with $100 \mu \mathrm{g}$ of the antigen suspension in $100 \mu$ l saline for 1 hour at $37^{\circ} \mathrm{C}$;

(2) addition of antigen to a culture chamber at a concentration of $10 \mu \mathrm{g} / \mathrm{ml}$, and into which untreated leucocytes were allowed to migrate.

Micropipette tubes, $6 \times 25 \mu$ l, were filled with the leucocyte suspensions, sealed with inert clay, spun at $50 \mathrm{~g}$ for 5 minutes, and cut at the cell fluid inter- face. Six tubes were prepared for each test and two were mounted in a spot of silicone grease in each of three disposable tissue culture chambers. The chambers were filled with Eagle's medium containing $10 \%$ FCS (and antigen if no preincubation had taken place), and incubated at $37^{\circ} \mathrm{C}$ for 18 to 24 hours.

Migration areas were drawn using a camera lucida attached to a light microscope, measured using a planimeter, and a mean of six readings was obtained for each test. The migration index was expressed as $\frac{\text { Mean area of migration with antigen }}{\text { Mean area of migration without antigen }}$.

All values outside the control mean \pm 2 SD were regarded as significant enhancement or inhibition.

\section{Results}

The overall results obtained are summarised in the Table. No significant effect was demonstrated in those experiments in which the antigens, Mallory bodies and normal liver, were added to the culture chambers. The results obtained with the preincubation technique using Mallory bodies as antigen are illustrated in the Figure. Seven of 17 patients with alcoholic hepatitis showed reactivity to Mallory bodies, a finding statistically significant (Fisher and Yates exact test) when compared with the controls (P < 0.01). With normal liver three out of 12 patients with alcoholic hepatitis showed reactivity, a result not significantly different from that of the controls $(P>0.05)$. Of the seven patients with alcoholic hepatitis who showed reactivity with Mallory bodies, only six were tested with normal liver, and one of these six gave a positive reaction.

The positive results obtained did not show any correlation with the clinical severity of the disease, but there was a trend suggesting that reactivity was associated with a more severe degree of alcoholic hepatitis based on the morphological assessment.

Results of leucocyte migration inhibition studies using Mallory bodies and a normal liver preparation in a control population and in patients with alcohol-induced liver disease

\begin{tabular}{|c|c|c|c|c|c|c|c|c|}
\hline & \multicolumn{4}{|c|}{ Preincubation } & \multicolumn{4}{|c|}{ Antigen added to culture chamber } \\
\hline & \multicolumn{2}{|l|}{$M B s$} & \multicolumn{2}{|c|}{ Normal liver } & \multicolumn{2}{|l|}{$M B s$} & \multicolumn{2}{|c|}{ Normal liver } \\
\hline & Inhib & Enhanc & Inhib & Enhanc & Inhib & Enhanc & Inhib & Enhanc \\
\hline $\begin{array}{l}\text { Controls } \\
\text { Fatty liver } \\
\text { Alcoholic hepatitis } \\
\text { Alcoholic cirrhosis }\end{array}$ & $\begin{array}{l}0 / 30 \\
0 / 6 \\
4 / 17 \\
2 / 15\end{array}$ & $\begin{array}{l}1 / 30 \\
1 / 6 \\
3 / 17 \\
2 / 15\end{array}$ & $\begin{array}{l}0 / 22 \\
0 / 4 \\
2 / 12 \\
1 / 12\end{array}$ & $\begin{array}{l}0 / 22 \\
0 / 4 \\
1 / 12 \\
0 / 12\end{array}$ & $\begin{array}{l}0 / 31 \\
0 / 4 \\
1 / 16 \\
1 / 14\end{array}$ & $\begin{array}{l}0 / 31 \\
0 / 4 \\
1 / 16 \\
1 / 14\end{array}$ & $\begin{array}{l}0 / 20 \\
0 / 3 \\
0 / 11 \\
1 / 8\end{array}$ & $\begin{array}{l}2 / 20 \\
0 / 3 \\
1 / 11 \\
0 / 8\end{array}$ \\
\hline
\end{tabular}

MBs = Mallory bodies (alcoholic hyalin).

Inhib = inhibition; Enhanc = enhancement.

* Number reacting/number tested. 


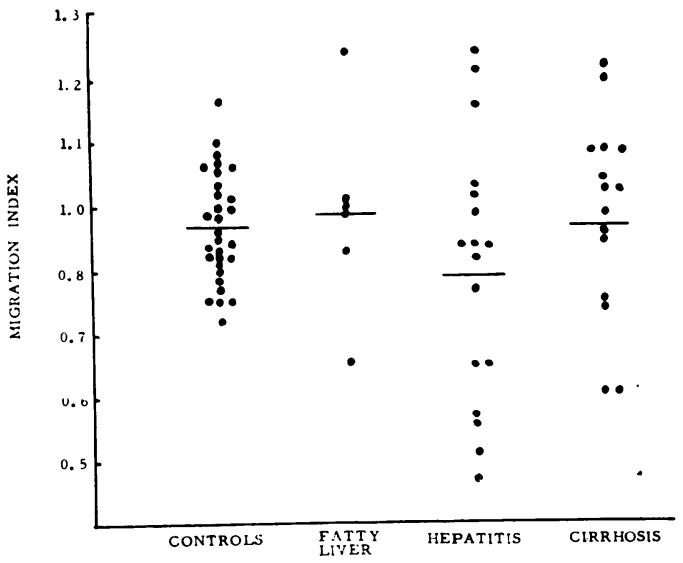

Migration indices for peripheral blood leucocytes pre-incubated with Mallory bodies (alcoholic hyalin) in normal control adults and in patients with alcohol-induced liver disease.

\section{Discussion}

The morphological features of alcoholic hepatitis comprise liver cell necrosis associated predominantly with a neutrophil polymorph reaction; the neutrophils may characteristically surround individual degenerate hepatocytes, or groups of neutrophils may invade damaged hepatocytes or may form discrete microaggregates. A variable lymphocytic and histiocytic infiltrate is also present. Cytologically, individual hepatocytes show hydropic or ballooning degeneration, and many contain irregular eosinophilic cytoplasmic aggregates, Mallory bodies, a characteristic although not specific feature of the disease. ${ }^{2}$ Fibrosis, initially pericellular in distribution, is an early accompaniment of the liver cell necrosis, and it is extension of this fibrosis that will ultimately produce hepatic cirrhosis. ${ }^{2}$

The precipitating factors in the development of alcoholic hepatitis, and the factors which lead to its progression to cirrhosis, remain controversial. The reported progression to cirrhosis, even after withdrawal of alcohol, ${ }^{411}$ suggests that mechanisms other than alcohol toxicity per se might be implicated. Immune mechanisms in particular have been postulated on the basis of a number of findings, including hyperglobulinaemia, in particular IgA, which may be elevated at an early change, ${ }^{14}$ low titre smooth muscle and antinuclear antibodies, ${ }^{15}$ a reversible impairment of delayed hypersensitivity, ${ }^{16}$ and a reduction in peripheral blood $\mathrm{T}$ lymphocytes. ${ }^{17}$

Evidence of sensitisation to liver antigens has also been sought. The lymphocytic infiltrate that occurs in the liver is predominantly $T$ cell, ${ }^{1819}$ and this contrasts with other forms of chronic liver disease. Furthermore, many of these $\mathrm{T}$ cells are apparently activated 19 and were found only during episodes of alcohol-induced hepatitis, findings which imply that $T$ lymphocytes have become sensitised to liver antigens and may be mediating a tissue-damaging reaction. The nature of the sensitising liver antigen(s) has not, however, been established. In vitro evidence of lymphocyte sensitisation to autologous liver was reported by Leevy and his colleagues on the basis of lymphocyte transformation and migration inhibition tests. ${ }^{6}$ In subsequent studies by the same group and using Mallory bodies isolated from the livers of patients with alcoholic hepatitis, they reported that lymphocytes sensitised to Mallory bodies produced migration inhibition factor ${ }^{7}$ and were cytotoxic for autologous liver cells. ${ }^{8}$ Furthermore, using complement fixation and immune adherence haemagglutination, they reported the demonstration of Mallory body antigens in the sera of patients with early phase alcoholic hepatitis, the antigen disappearing within three to five weeks of abstinence from alcohol. ${ }^{20}$ This antigen was demonstrable in only a minority of patients with advanced alcoholic hepatitis, in none with alcoholic fatty liver or inactive cirrhosis, and in none of a group of patients with various other forms of acute liver disease. Antibody to Mallory bodies was found in the sera of all patients with advanced alcoholic hepatitis, in a small minority of patients with early phase alcoholic hepatitis, and in none of the other groups examined. ${ }^{20}$ The antibody also disappeared with abstinence from alcohol and clinical subsidence of alcoholic hepatitis. Immunoglobulin eluted from liver homogenates of patients with advanced alcoholic hepatitis and active cirrhosis were found to contain antibodies to Mallory-body antigen of both IgA and IgG class. ${ }^{20}$ These findings suggest that immunological reactivity to Mallory bodies may be of key importance in producing the liver cell damage that occurs in alcoholic hepatitis.

The studies that we report here were undertaken in an attempt to confirm some of the findings of Leevy and his colleagues. We found that Mallory bodies were easily isolated from postmortem livers. However, these Mallory bodies were sparingly soluble in a number of agents-guanidine buffer, ${ }^{7}$ $0.1 \%$ tris buffer, ${ }^{20}$ and $1 \%$ sodium dodecyl sulphate ${ }^{21}$-and, furthermore, in control migration inhibition tests these solvents were toxic for leucocytes and inhibited their migration. Because of this, sonicated suspensions of Mallory bodies were used in our studies, a treatment which resulted in the antigen being present in a particulate form.

In tests in which untreated leucocytes migrated into a culture medium containing Mallory body 
antigen, we were unable to demonstrate any inhibitory effect, a result which may have been due to an insufficient antigen concentration. In contrast, however, when the antigen was reacted directly with leucocytes for 1 hour before their use in migration inhibition tests, in vitro sensitisation was demonstrable in a statistically significant $41 \%$ of patients with alcoholic hepatitis. The antigen used in the tests was not absolutely pure, and the demonstrated sensitisation to 'normal-liver antigen' in three of 12 patients with alcoholic hepatitis also raises the possibility of sensitisation to other liverderived antigens in these patients. Current evidence, however, suggests that Mallory bodies comprise intermediate filaments, constituents of normal hepatocytes, which accumulate in the alcoholdamaged hepatocyte because of the anti-microtubule action of alcohol. ${ }^{13}$ Thus, the results obtained with 'normal-liver antigen' may reflect the sensitisation to Mallory bodies or Mallory bodylike material.

Our results thus provide some support for the findings of Leevy and his colleagues ${ }^{j}$ and for their claim that sensitisation to Mallory bodies may be of significance in the pathogenesis of alcoholic hepatitis. If chronic alcohol ingestion produces lymphocyte sensitisation to a liver cell antigen, then lymphocyte-hepatocyte interaction may cause hepatocyte necrosis. Further studies are now being undertaken to test this postulate.

\section{References}

${ }^{1}$ Mowat NAG, Brunt PW. Alcohol and the gastrointestinal tract. Recent Advances in Gastroenterology 1976;3:150-77.

${ }^{2}$ MacSween RNM. Alcoholic liver disease. Recent Advances in Histopathology 1978;10:193-212.

${ }^{3}$ Lelbach WK. Quantitative aspects of drinking in alcoholic liver cirrhosis. In: Khanna JM, Israel Y, Kalant H, eds. Alcoholic Liver Pathology. Toronto: Addiction Research Foundation of Ontario, 1975:1-18.

+ Galambos JT.The course of alcoholic hepatitis. In: Khanna J M, Israel Y, Kalant H, eds. Alcoholic Liver Pathology. Toronto: Addiction Research Foundation of Ontario, 1975:97-111.

${ }^{5}$ Leevy CM, Chen T, Luisada-Opper A. Liver disease of the alcoholic: role of immunologic abnormalities in pathogenesis, recognition and treatment. In: Popper $\mathrm{H}$, Schaffner F, eds. Progress in Liver Diseases Vol 5. New York: Grune Stratton, 1976:516-30.
${ }^{6}$ Zetterman RK, Leevy CM. Immunologic reactivity and alcoholic liver injury. Bull N Y Acad Med 1975;51 :533-44.

- Zetterman RK, Luisada-Opper A, Leevy CM. Alcoholic hepatitis: cell mediated immunologic response to alcoholic hyalin. Gastroenterology 1976:70:382-4.

" Kakumu S, Leevy CM. Lymphocyte cytotoxicity in alcoholic hepatitis. Gastroenterology 1977;72:594-7.

${ }^{9}$ Hardison WG, Lee FI. Prognosis in acute liver disease of the alcoholic patient. $N$ Engl J Med 1966;275:61-6.

${ }^{10}$ Lischner MW, Alexander JF, Galambos JT. Natural history of alcoholic hepatitis. I: The acute disease. $\mathrm{Am} \mathrm{J}$ Dig Dis 1971;16:481-94.

${ }^{11}$ Brunt PW, Kerr MC, Scheuer PJ, Sherlock S. Studies in alcoholic liver disease in Britain. Gut 1974;15:52-8.

${ }^{12}$ Okamura K, Harwood TR, Yokoo H. Isolation and electrophoretic study on Mallory bodies from the livers of alcoholic cirrhosis. Lab Invest 1975;33:193-9.

${ }^{13}$ French SW, Sim JS, Caldwell MG. Thick microfilaments (intermediate filaments) and chronic alcohol ingestion. In: Popper H, Bianchi L, Reutter W, eds. Membrane Alterations as a Basis of Liver Injury. Lancaster: MTP Press Ltd, 1976:311-25.

${ }^{14}$ Morgan et al. cited in Thomas HC, Jewell DP, eds. Clinical Gastrointestinal Immunology. London: Blackwell Scientific Publications, 1979:242.

${ }^{15}$ Bailey RJ, Krasner N, Eddleston ALWF, Williams R, Tee DEH, Doniach D, Kennedy LA, Batchelor JR. Histocompatibility antigens, autoantibodies and immunoglobulins in alcoholic liver disease. $\mathrm{Br} \mathrm{Med} J$ 1976;2:727-9.

${ }^{16}$ Berenyi MR, Straus B, Cruz D. In vitro and in vivo studies of cellular immunity in alcoholic cirrhosis. Amer J Dig Dis 1974;19:199-209.

${ }^{17}$ Bernstein IM, Webster KA, Williams RC, Strickland RG. Reduction in T lymphocytes in alcoholic liver disease. Lancet 1974;2:488-90.

${ }^{18}$ Husby G, Strickland RG, Caldwell JL, Williams RC. Localisation of $\mathrm{T}$ and $\mathrm{B}$ cells and alpha fetoprotein in hepatitis biopsies from patients with liver disease. $J$ Clin Invest 1975;56:1198-209.

19 Sanchez-Tapias J, Thomas HC, Sherlock S. Lymphocyte populations in liver biopsy specimens from patients with chronic liver disease. Gut 1977;18:472-5.

${ }^{20}$ Kanagasundaram N, Kakumu S, Chen T, Leevy CM. Alcoholic hyalin antigen (AHAg) and antibody (AHAb) in alcoholic hepatitis. Gastroenterology 1977;73:1368-73.

${ }^{21}$ Leevy CM, Kanagasundaram K, Matsumoto K, Chen T. Alcoholic hyalin and immunologic reactivity. In: Eddleston ALWF, Weber JCP, Williams R, eds. Immune Reactions in Liver Disease. London: Pitman Medical, 1979:195-207.

Requests for reprints to: Professor RNM MacSween, Pathology Department, University of Glasgow, Western Infirmary, Glasgow G11 6NT. 